\title{
ALGORITHMS TO GET A CIRCULATING BEAM
}

\author{
AndrêAVerdier, Frank Richard \\ CERN CH1211 Geneva 23
}

\begin{abstract}
Two algorithms based of trajectory $®$ tting have been used to obtain rapidly a circulating beam in LEP with strong focusing lattices.

A new algorithm called arbit closure ${ }^{\mathrm{o}}$ uses the beam positions measured at the $B$ rst and the second turn. From their difference, it computes two corrector strengths per plane such that the second turn passes at the same place as the ${ }^{\circledR}$ rst turn at about ten BPM locations. This produces a closed orbit amplitude with an r.m.s. value close to that of the ®rst turn trajectory.

A new '®rst turn threader' has been tried. It detects places where an oscillation with an r.m.s. amplitude larger than a speci®ed value starts, and computes two corrector strengths to cancel it. By iterating this process, it should be possible to correct by steps the ${ }^{\circledR}$ rst turn trajectory so that its r.m.s. amplitude is below a speci®ed value.
\end{abstract}

The orbit closure has been applied with success to a very low emittance lattice $\left(135^{\circ}\right.$ in the horizontal plane) in LEP.

\section{The principles}

These algorithms are based on the $\AA$ tting method. This method assumes that the linear optics of the machine is well known. It relies on the description of betatron oscillations with an expression like :

$$
y_{i}=a \sqrt{\beta_{i}} \cos \left(\mu_{i}\right)+b \sqrt{\beta_{i}} \sin \left(\mu_{i}\right)+c+\eta_{i}
$$

where $y_{i}$ stands for the $i^{\text {th }}$ measurement of the beam position, $\beta_{i}$ and $\mu_{i}$ for the corresponding TWISS parameters and $\eta_{i}$ for a realization of a null-mean additive noise. Given this deßnition of $\eta_{i}$, we must put in the equation the average of the noise which is $c$. This parameter can be interpreted also as an offset of the measurements.

The values of $a, b$ and $c$, are computed by means of the leastsquares algorithm. In fact what is interesting is not the values of $a, b$ or $c$ themselves, but how relevant is the ${ }^{\circledR} \mathrm{t}$, i.e. whether the measurements follow a betatron oscillation or not. To answer this question, the residual $F_{n}$ of the ${ }^{\circledR} \mathrm{t}$ done with $n$ measurements is computed. Its expression is :

$$
F_{n}=\sqrt{\frac{1}{n-3} \sum_{i=1}^{n}\left(\tilde{y}_{i}-y_{i}\right)^{2}}
$$

where $\tilde{y_{i}}$ is the estimation of $y_{i}$ obtained once the constants $a, b$ and $c$ have been computed.

A very important feature of the @tting method is the possibility to discard the measurements considered as bad, i.e. those such that $\left|\tilde{y}_{i}-y_{i}\right|$ is larger than a certain number of standard deviations. Usually this number is between two and three as we discard measurements because they are at the edge of a normal distribution of random variables. Note that the best estimate of the standard deviation is $F_{n}$. Once the bad measurements have been discarded, the computation is remade until no measurement is considered as bad.

\section{Orbit closure}

\section{A. The algorithm}

The ${ }^{\circledR}$ rst step for operating a circular machine is to get a ${ }^{\circledR}$ rst turn. However this usually does not guaranty that the beam circulates because the closed orbit distortion may be too large. Furthermore, in the case of strong focusing lattices, the chromaticity sextupoles are strong because the dispersion is small and this makes large anharmonicities which can make a linear instability if one of the tunes comes close to an integer. This may well happen for a closed orbit distortion which would not prevent the beam to circulate with a weak focusing lattice. This is precisely the case where the orbit closure algorithm is the most useful.

When a ${ }^{\circledR}$ rst turn is obtained in a machine, the beam position can be measured at several places close to the point where it enters the machine, both during the ${ }^{\circledR}$ rst and the second turn. Then the ®tting method is applied to the difference between these beam positions, and this difference is canceled out with two closed orbit correctors in each plane. Once it is made small enough, it is possible to obtain a circulating beam.

Practically twenty positions are measured downstream of the place where the injected beam enters LEP. After elimination of wrong measurements, only ten which are considered good from the ${ }^{\circledR} t$ ting of the difference between $\circledR$ rst and second turn are kept for the computation. It is worth noting that the BPM's which suffer from a mere offset are not considered as bad usually.

\section{B. Experience with a strong focusing lattice}

An illuminating example of the ef®ciency of the closure algorithm is provided by a machine experiment done with a lowemittance lattice in LEP. The LEP machine at CERN is made from eight arc, each containing thirty FODO cell with dipoles and sextupoles, and eight insertions which contain only dipoles and quadrupoles. The super-periodicity is four. All the nonlinear elements (sextupoles) are in the arc cells. Low-emittance lattices have been envisaged for LEP as a mean to increase the luminosity at low current [1]. In one of these lattices, which was found to be dif®cult to operate, the arc FODO cells have an horizontal phase advance of $135^{\circ}$ and a vertical phase advance of $60^{\circ}$. The horizontal tune is 125.28 and the vertical tune is 75.18 . Given the machine super-periodicity, such tunes guaranty that no problem is expected from the non-linear chromaticity [4].

During year 1993, two eight hours shifts had been done where no circulating beam was obtained. This led us to an extensive analysis of the problems associated with this lattice in order to understand why it was so dif®cult. A report on this study is available [2] and some of the results concerning this lattice can be found at this conference [1]. One interesting outcome of this 
study was that it is not possible to correct both third order resonances and horizontal anharmonicity. It was felt that it was easier to try to cope with the anharmonicity than with the third order resonances. The horizontal anharmonicity, i.e. the derivative of the horizontal tune with respect to the horizontal emittance, has a value of $-1.6 \times 10^{5} \mathrm{~m}^{-1}$ for the lattice under consideration. For an r.m.s. closed orbit amplitude of 4mm at the BPM's where $\beta_{x}$ is about $10 \mathrm{~m}$, the associated emittance is about $1.6 \mu \mathrm{rad} . \mathrm{m}$, which makes an associated horizontal tune-shift is -0.26 . As the fractional part of the horizontal tune of our machine is 0.28 , we see that a badly corrected closed orbit can easily lead to an horizontal instability due to the fact that the horizontal tune becomes an integer. In practice it can be observed that the horizontal tune, estimated from a Fourier analysis of several turns measurements, wanders considerably depending on the corrections applied to the $®$ rst turn trajectory.

In the successful experiment, a great care was taken ®rst to correct the $®$ rst turn trajectory so that its r.m.s. value was between 3 and $2 \mathrm{~mm}$. This took about $®$ ve hours and this is why a more powerful algorithm, described in the next section, was studied. When the ®rst turn was corrected, the orbit closure algorithm was applied but no circulating beam was obtained although the goal was not far, according to the signal of the RF pick-up indicating that some particles were trapped in the RF buckets for several turns. It took us some time to realize that in fact corrector increments of the order of $10 \mu \mathrm{rad}$ had to be applied. Indeed usually closed orbit corrections of this importance are not vital as a kick of $10 \mu \mathrm{rad}$ at a place where $\beta_{x}$ is $10 \mathrm{~m}$ makes a closed orbit distortion of less than $0.1 \mathrm{~mm}$ if the fractional part of the tune is close to 0.3 . In the present case, if the ®rst turn trajectory has an r.m.s. amplitude above $3 \mathrm{~mm}$, the horizontal anharmonicity brings the horizontal tune close to an integer, which in turn ampli®es the closed orbit distortion due to this small kick an makes the betatron oscillations unstable.

\section{First turn threader}

\section{A. Why a new threader?}

A new algorithm is in the process of being developed. In fact there is already a usable facility in LEP, but it is extremely lengthy to make a small r.m.s. oscillation amplitude. In order to develop more easily strong focusing lattices, a faster automatic algorithm, which produces a $®$ rst turn trajectory with an r.m.s. deviation as small as possible, is needed as mentioned above.

Before a ${ }^{\circledR}$ rst turn is done, the injected beam is steered as well as possible, so that its oscillation amplitude just after its entrance in the machine can be reduced to some tenths of millimeters. However as soon as it encounters ®eld defects, the oscillation amplitude increases and the beam is eventually lost.

The idea underlying the new threader was to detect these growing oscillations and to correct them one after the other using a single trajectory measurement. In this process, after a correction is computed, its contribution is subtracted from the trajectory measurement and the next correction is computed with the corrected trajectory. It is clear that, if the beam is lost in the $®$ rst turn, this can only be partially applied and this algorithm is not valid close to the places where the beam is lost. The aim was in fact to improve a ${ }^{\circledR}$ rst turn trajectory from a full turn measure- ment.

\section{B. What has been achieved}

The $®$ rst part of the new process was to establish a simple algorithm to detect $® e l d$ defects. The algorithm is similar to that used for the defect search from a closed orbit measurement [3], but simpler. A $\cap t$ of the measured trajectory was done from the injection point starting at each BPM and the residue of each ®t was computed. A threshold was set for these residues, above which it was considered that a defect must be there.

This looks simple minded but in practice, it is not possible to determine the location of discontinuities in the measured trajectory by merely looking at the ${ }^{\circledR}$ rst large residue and deciding that the defect lies close to the last BPM used to do the $®$ t. It was noticed that, instead of this, the detected defect was close to the $®$ rst BPM used to do the ®t. As ten BPM's are used, this can make quite a difference in the location! Once the location of a defect is found, even approximately, two closed orbit correctors upstream of the detected defect are chosen to cancel the downstream oscillation. This complete procedure which consists of identifying defects and making corrections was the main outcome of the ®rst study of the algorithm in the LEP control system.

The next step of the algorithm was to compute a correction for several successive defects. After a ßrst defect has been found, a correction is computed and its contribution to the oscillation is subtracted from the measurements. Then the next defect is sought and the process is iterated. Applying this in practice shown us that it was not possible to go further than about four defects because the algorithm is linear and the machine is not. Another complication came from the existence of the experimental solenoids which make a coupling between the two oscillation planes. Although this can be handled with a formalism based on $4 \times 4$ transfer matrices, this is not yet available in the LEP control system. Thus the only possibility is to enter the coupled sections with very low oscillation amplitudes. This was not done in the program we used, therefore the algorithm was not successful.

Nevertheless the two above conclusions are already extremely useful to carry on a study on a fast threader for strong focusing lattices.

\section{Conclusion}

The orbit closure algorithm, which was already recommended for the LEP startup in 1989, proved to be extremely useful to obtain a circulating beam with a very strongly focusing lattice.

Devising a fast threader algorithm is far from being an easy task. In fact our study is only at its starting stage and we arrived already at useful recommendations to develop it.

\section{References}

[1] Y. Alexahin, D. Brandt, K. Cornelis, A. Hofmann, J.P. Koutchouk, M. Meddahi, G. Roy, A. Verdier, Low emittance lattice for LEP. This conference.

[2] Y. Alexahin,

A study of the low emittance lattice for LEP2. CERN SL/9446(AP). Divisional report. 
[3] A. Verdier and J.C. Chappelier, An automatic finder of field defects in large A.G. machines. PAC Washington D.C. 1993.

[4] A. Verdier,

Higher order tune derivatives due to low- $\beta$ insertions. 1993

Part. Acc. Conf., Washington D.C., 17-20 May 1993. 